\title{
Species-dependent differences of embryonic stem cell-derived neural stem cells after Interferon gamma treatment
}

\author{
Janine Walter ${ }^{1,2 *}$ and Marcel Dihné ${ }^{2}$ \\ 1 Department of Neurology, Heinrich-Heine-University Düsseldorf, Düsseldorf, Germany \\ 2 Department of Neurology and Epileptology, Hertie Institute for Clinical Brain Research, Eberhard-Karls-University, Tübingen, Germany
}

Edited by:

Eran Meshorer, The Hebrew University of Jerusalem, Israel

\section{Reviewed by:}

Marcel Leist, University

of Konstanz, Germany

Yuanquan Song, University

of California San Francisco, USA

\section{*Correspondence:}

Janine Walter, Department

of Neurology, Heinrich-Heine-

University, Moorenstr. 5,

40225 Düsseldorf, Germany.

e-mail: janine.walter@

googlemail.com
Pluripotent stem cell (pSC)-derived, neural stem cells (NSCs) are actually extensively explored in the field of neuroregeneration and to clarify disease mechanisms or model neurological diseases in vitro. Regarding the latter, proliferation and differentiation of pSC-derived NSCs are investigated under the influence of a variety of different substances among them key players of inflammation. However, results generated on a murine genetic background are not always representative for the human situation which increasingly leads to the application of human cell culture systems derived from human pSCs. We investigated here, if the recently described interferon gamma (IFN $\gamma$ )-induced dysregulated neural phenotype characterized by simultaneous expression of glial and neuronal markers on murine NSCs (Walter et al., 2011, 2012) can also be found on a human genetic background. For this purpose, we performed experiments with human embryonic stem cell-derived NSCs. We could show that the IFN $\gamma$-induced dysregulated neural phenotype cannot be induced in human NSCs. This difference occurs, although typical genes like signal transducers and activators of transcription 1 (Stat 1) or interferon regulatory factor 9 (IRF-9) are similarly regulated by IFN $\gamma$ in both, murine and human populations. These results illustrate that fundamental differences between murine and human neural populations exist in vitro, independent of anatomical system-related properties.

Keywords: human neural stem cells, inflammation, interferon-gamma, neurodegeneration, IFN $\gamma$

\section{INTRODUCTION}

Neural stem cells (NSCs) are multipotent and can give rise to the three principle cell types found in the adult mammalian brain, namely neurons, astrocytes, and oligodendrocytes. In vivo, they can be found in the hippocampus and subventricular zone where they mainly give rise to new neurons in the dentate gyrus and olfactory bulb, a process called neurogenesis (Kempermann, 2002, 2011). In vitro, they can be kept under proliferative conditions exhibiting immature neural markers like nestin and Sox2 due to the stimulus of growth factors, or differentiate to the above mentioned mature cell types when growth factors are withdrawn (Temple and Qian, 1996; Temple, 2001). Various regenerative approaches are aimed at using the capacity of NSCs as a therapeutic agent by transplanting them into injured brain regions (Barker et al., 2003; Harrower and Barker, 2005; Gogel et al., 2011). Additionally, NSCs from various sources are increasingly used to simulate diseases in vitro in order to set up model systems that can easily be manipulated and investigated. A specific and important advantage of in vitro model systems for neurological diseases is the possibility to use a human genetic background on the basis of human pluripotent stem cells (pSCs) in order to exclude, for instance, murine-specific phenomena. One interesting question, that could be clarified in vitro under controlled conditions, is how inflammatory processes that consistently occur in various neurological diseases act on NSCs. As in vivo neurogenesis is more and more accepted to contribute to processes like, for instance, memory function (Deng et al., 2009, 2010), it appears to be of interest to characterize effects of pathological processes such as inflammation on NSCs. Inflammation itself may lead to damage in brain tissue and represents either a primary disease entity or a secondary phenomenon following (Campbell, 2005), for instance, cerebral ischemia (Whitney et al., 2009). Interferon gamma (IFN $\gamma$ ), a pro-inflammatory key player, is a cytokine that is secreted by various cell types such as cytotoxic CD8 ${ }^{+}$ T-cells, natural killer cells (Griffin, 1997), astrocytes, fibroblasts, and endothelial cells (Rady et al., 1995; De Simone et al., 1998; Wei et al., 2000). IFN $\gamma$ signaling takes place via the IFN $\gamma$ receptor which consists of two chains, situated in the cell membrane with an extra- and intracellular part (Schreiber and Farrar, 1993). The structure and the amino acid sequence of the murine and the human IFN $\gamma$ protein and its receptor differ, although the physiological function remains the same (Farrar and Schreiber, 1993). These structural differences are leading to species-specific IFN $\gamma$-IFN $\gamma$ receptor interactions with human IFN $\gamma$ affecting only human and other primate cell types and vice versa (Schreiber et al., 1992; Schroder et al., 2004). IFN $\gamma$ receptors were found on murine NSCs and therefore, effects of IFN $\gamma$ on murine NSCs and related alterations in neurogenesis in vivo (Kim et al., 2002; Lin et al., 2004; Sweeten et al., 2004; Wang et al., 2004, 2008) and in vitro (Kim et al., 2007; Makela et al., 2010) were excessively 
explored. However, only little is known about the response of human NSCs (hNSCs) to IFN $\gamma$. We previously investigated effects of IFN $\gamma$ on murine embryonic day 14-derived stem/precursor cells (msNSPCs) and murine embryonic stem cell-derived NSCs in vitro (Walter et al., 2011, 2012) (both populations are referred to as mNSCs in the following text). Predominantly in proliferative mNSC cultures, we found that IFN $\gamma$ leads to a dysregulated phenotype, characterized by synchronous expression of neuronal and glial markers despite the presence of growth factors. This was accompanied by an unusual electrophysiological phenotype on single cell level preventing the ability to form synchronously bursting functional neuronal networks after differentiation. We also demonstrated an IFN $\gamma$-related significant up-regulation of sonic hedgehog $(\mathrm{SHH})$ and Stat 1, key down-stream signals that are important for induction of the above mentioned phenotype (Walter et al., 2012). To assess the relevance of these findings with respect to the human situation, we (1) treated human embryonic stem cell-derived hNSCs with this pro-inflammatory cytokine and (2) measured IFN $\gamma$ concentrations in cerebrospinal fluid (CSF) specimens collected from patients suffering from different nervous system diseases.

\section{RESULTS}

\section{hNSCs EXPRESS IFN $\gamma$ RECEPTOR I AND II}

In a first step, we immunocytochemically characterized the hNSC population generated from immature pluripotent embryonic stem cells. After neural pre-differentiation, almost all cells expressed nestin and most cells (>80\%) expressed both, nestin and Sox2 (Figure 1A). After withdrawal of bFGF, NSCs terminally differentiated into $\beta$ III-tubulin ${ }^{+}$neurons or $\mathrm{GFAP}^{+}$astrocytes (Figure 1A). As the expression of membrane-bound IFN $\gamma$ receptors ( 2 IFN $\gamma$ receptor- 1 chains and 2 IFN $\gamma$ receptor- 2 chains) is necessary to transduce the IFN $\gamma$ signal, we performed immunocytochemical labelings against both receptor chains and were indeed able to demonstrate their expression (Figure 1B). We further investigated the expression levels of both receptor chains on mRNA level by means of real-time quantitative PCR. We compared these data with those generated on a murine background and found, that murine and human NSCs did not show significant differences (Figure 1C). Data were generated with the Wicell H9 line. We also confirmed these findings by using the HUES 6 line (data not shown).

\section{hNSCS DO NOT EXPRESS THE DYSREGULATED PHENOTYPE AFTER IFN $\gamma$ EXPOSURE}

One major characteristic of the IFN $\gamma$-induced mNSC dysregulation is the coexpression of neuronal and glial markers under the influence of growth factors that normally hold NSCs in an immature and proliferating state (Figure 2A). This phenomenon is visible after a 3-days treatment with $1000 \mathrm{U} / \mathrm{ml}$ IFN $\gamma$ and leads to a portion of around 39\% of all cells that co-express GFAP and $\beta I I I-t u b u l i n$. The detailed characterization of this phenomenon is published elsewhere (Walter et al., 2011). However, when immature and proliferating hNSC populations under the influence of growth factors were treated with human recombinant IFN $\gamma$ in identical concentrations compared to the murine situation, we were not able to detect this phenomenon (Figure 2A). These

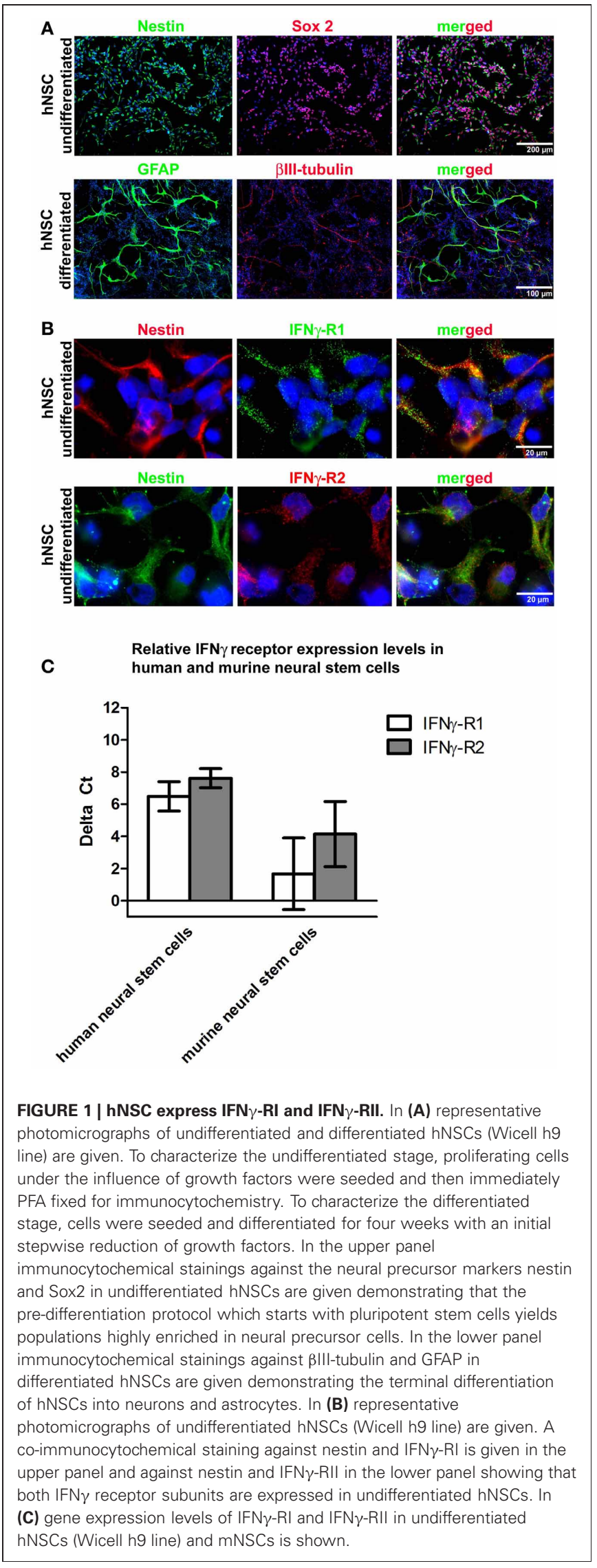

B
C

FIGURE 1 | hNSC express IFN $\gamma$-RI and IFN $\gamma$-RII. In (A) representative photomicrographs of undifferentiated and differentiated hNSCs (Wicell h9 are given. To characterize the undifferentiated stage, proliferating cells PFA fixed for immunocytochemistry. To characterize the differentiated stage, cells were seeded and differentiated for four weeks with an initial stepwise reduction of growth factors. In the upper panel and Sox2 in undifferentiated hNSCs are given demonstrating that the immunocytochemical stainings against $\beta / I$-tubulin and GFAP in differentiated hNSCs are given demonstrating the terminal differentiation ons into neurons and astrocytes. In (B) representative upper panel and against nestin and IFN $\gamma$-RII in the lower panel showing that (C) gene expression levels of IFN $\gamma$-RI and IFN $\gamma$-RII in undifferentiated hNSCs (Wicell h9 line) and mNSCs is shown. 


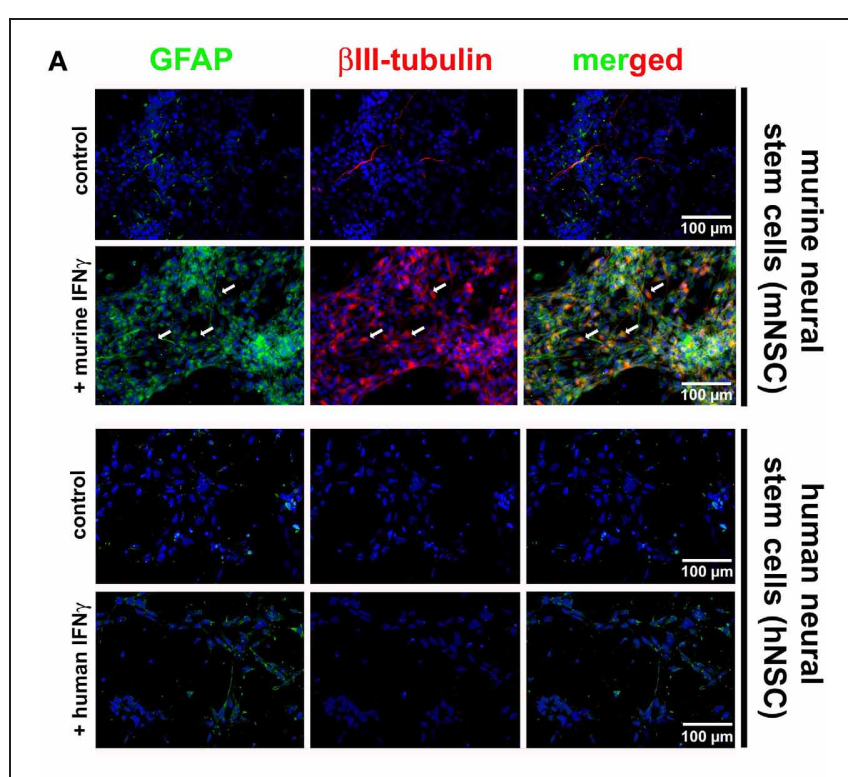

gene expression levels in
B $\quad$ undifferentiated hNSC

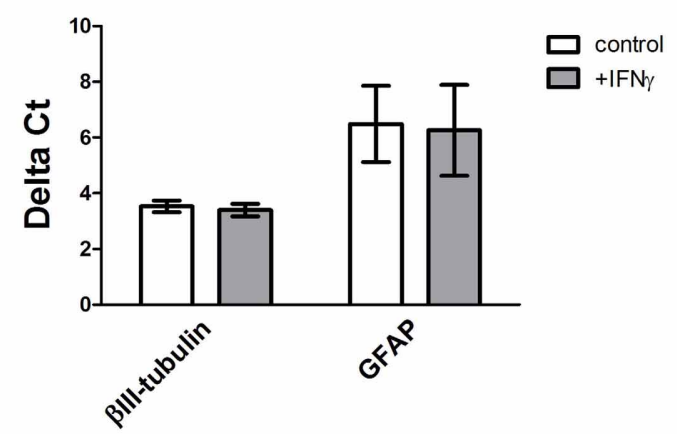

FIGURE 2 | Effects of IFN $\gamma$ on hNSCs and mNSCs. In (A) photomicrographs of undifferentiated hNSCs (Wicell h9 line) and mNSCs are given. A $\beta$ III-tubulin and GFAP co-staining under control conditions and after IFN $\gamma$ treatment is shown. The upper to panels show mNSCs, the lower two panels show hNSCs. In mNSCs, IFN $\gamma$ typically leads to a strong up-regulation of neuronal ( $\beta$ III-tubulin) and glial (GFAP) markers with many cells simultaneously expressing both markers (arrows and the merged picture). In hNSCs no IFN $\gamma$-induced up-regulation of these markers was detected. In (B) gene expression levels of $\beta$ III-tubulin, GFAP, and $\mathrm{SHH}$ in undifferentiated hNSCs (Wicell h9 line) either under control conditions or after IFN $\gamma$ treatment are shown. The results demonstrate that there is, also on mRNA level, no IFN $\gamma$-induced up-regulation of cell type-specific markers.

results were also confirmed on mRNA level (Figure 2B). Data were generated with the Wicell $\mathrm{H} 9$ line. We also confirmed these findings by using the HUES 6 line (data not shown).

\section{IFN $\gamma$-REGULATED GENES IN hNSCs AND EFFECTS OF IFN $\gamma$ ON THE POPULATION SIZE OF hNSCs}

As previously reported, the genes for inducible nitric oxid synthase (i-NOS) (Komatsu et al., 1996), IRF-9 (Ousman et al., 2005), c-Myc (Ramana et al., 2000), major histocompatibility complex 1 (MHC 1) (Johansson et al., 2008) and Stat 1 (Lehtonen et al., 1997) are significantly regulated in murine cells after IFN $\gamma$ exposure. We now investigated the expression of these downstream signaling genes after IFN $\gamma$ exposure in hNSCs by means of real-time quantitative PCR. We found all of these genes to be significantly up-regulated after IFN $\gamma$ exposure also in hNSCs (Figure 3A). As previously reported, we also found $\mathrm{SHH}$ to be significantly up-regulated in mNSCs after IFN $\gamma$ treatment. Interestingly, we could not detect that phenomenon in hNSCs. Another IFN $\gamma$-induced phenomenon on a murine background was a strong reduction of the population size of undifferentiated mNSCs. However, we were not able to detect a significant decrease in the population size of hNSCs after IFN $\gamma$ exposure (Figure 3B). Data were generated with the Wicell H9 line. We also confirmed these findings by using the HUES 6 line (data not shown).

\section{IFN $\gamma$ IS UP-REGULATED IN THE CEREBROSPINAL FLUID OF PATIENTS SUFFERING FROM DIFFERENT DISEASES}

In contrast to the extensively described up-regulation of IFN $\gamma$ in animal models of human central nervous system diseases, there is only very limited information about amounts of IFN $\gamma$ in human individuals suffering from neurological diseases. Therefore, we measured the concentration of IFN $\gamma$ in cerebrospinal fluid (CSF) specimens, taken after lumbar punction from patients suffering from multiple sclerosis, peripheral nervous plexus affections, Alzheimer dementia, viral myelitis, or stroke. We found, that patients suffering from multiple sclerosis during an acute relapse showed a significant increase of IFN $\gamma$ (three control samples vs. three MS samples). Due to the limitation of patient material, we could only screen single patients with other diseases (one patient per other indicated disease). But also here we found a clear trend toward an up-regulation of IFN $\gamma$ in the CSF (Figure 3C).

\section{DISCUSSION}

We found here that IFN $\gamma$ has species-dependent effects on embryonic stem cell-derived neural populations. While this proinflammatory key player induces a striking dysregulation in undifferentiated murine neural stem cells (mNSCs) with an unusual coexpression of neuronal and glial markers (Walter et al., 2011, 2012), it has no such effects on human NSCs although both cell populations express appropriate IFN $\gamma$ receptors and upregulate most of the classical down-stream signals like i-NOS, IRF-9, c-Myc, MHC 1, and Stat 1, which are known to mediate important IFN $\gamma$ effects (Schroder et al., 2004).

One possible explanation for this diverse reaction of murine or human NSCs toward IFN $\gamma$ exposure might be subtle differences in their developmental state or brain region-specific differentiation although both populations consist of proliferating nestinand Sox2-positive neural precursors. However, the differentiation protocols used in this study were similar for both species leading to a heterogeneous population of neural cells without preference of distinct brain-region specific phenotypes (Illes et al., 2009; Lappalainen et al., 2010; Yla-Outinen et al., 2010). To account for developmental differences, we tested additional IFN $\gamma$ treatment paradigms for human NSCs including a 7-days treatment or a 5 -fold IFN $\gamma$ concentration but could not detect an up-regulation of neuronal or glial markers or even the appearance of cells that synchronously express these markers (data not shown). 


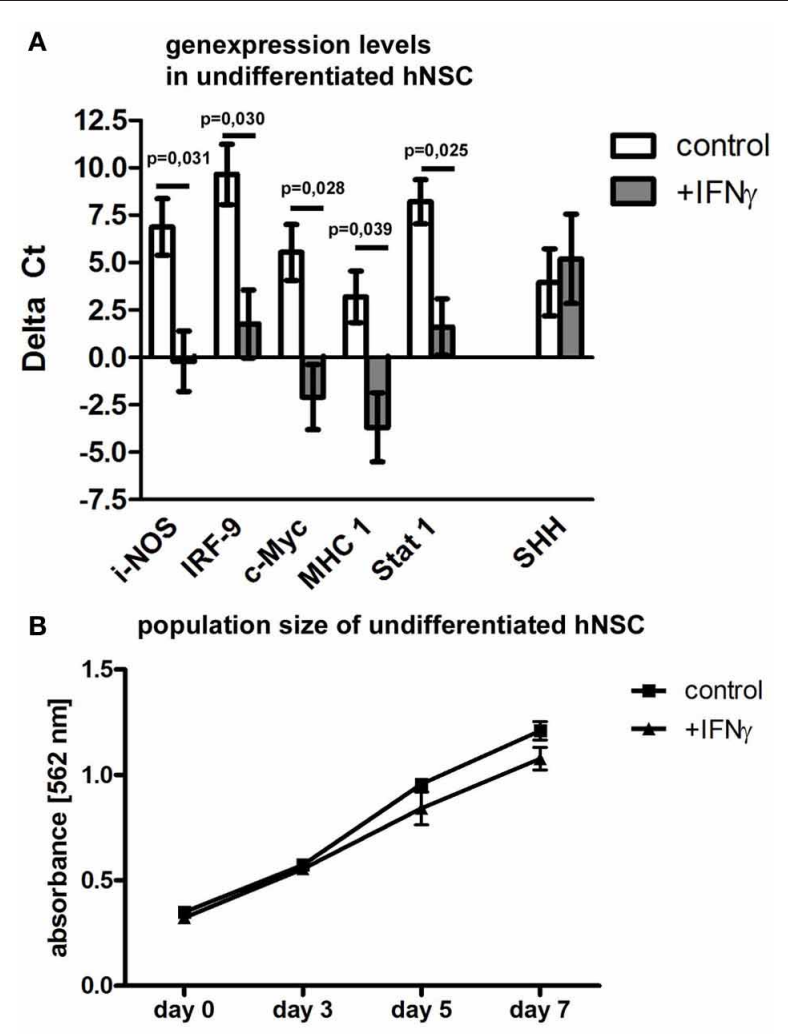

C amounts of IFN $\gamma$ in human CSF samples

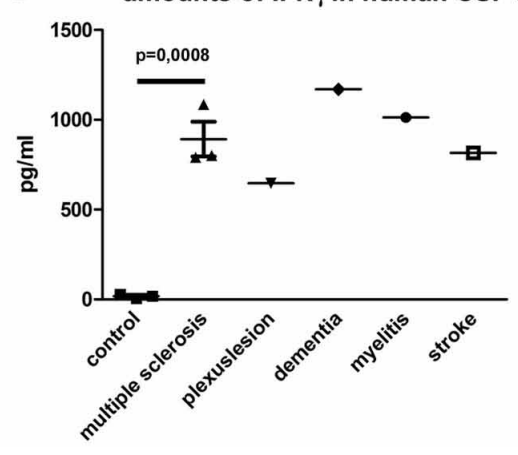

FIGURE 3 | Down-stream gene regulation and population size of hNSCs after IFN $\gamma$ exposure and in vivo levels of IFN $\gamma$ in patients CSF samples. In (A) gene expression levels in hNSCs (Wicell h9 line) of i-NOS (inducible nitric oxid synthase), IRF-9 (interferon regulatory factor 9), c-Myc, MHC1 (major histocompatibility complex 1), and Stat 1 (signal transducers and activators of transcription 1) are given either under control conditions or after IFN $\gamma$ treatment. Results show that IFN $\gamma$ significantly up-regulates important down-stream factors except of $\mathrm{SHH}$. Values are means \pm standard error of mean (SEM). Experiments were performed in triplicate and repeated independently at least three times. In (B) population size of hNSCs (Wicell h9 line) over 7 days is measured by means of an MTT-assay. Population sizes of two groups, hNSCs under control conditions or after IFN $\gamma$ treatment, are compared. Results show that IFN $\gamma$ does not affect the population size of hNSCs. Values are means \pm standard error of mean (SEM). Experiments were performed in triplicate and repeated independently at least three times. In (C) amounts of IFN $\gamma$ in clinical CSF samples are measured by ELISA in comparison to CSF samples of control patients. For statistical testing of the multiple sclerosis group, 3 control patients were compared with 3 multiple sclerosis patients [unpaired two-tailed $t$-test, value is mean \pm standard error of mean (SEM)].
Another explanation for the divergent reactions after IFN $\gamma$ exposure might be a qualitatively different induction of downstream signals. While $\mathrm{SHH}$ is significantly up-regulated after IFN $\gamma$ treatment in mNSC populations, it remains unaltered in hNSC populations. Interestingly, recently published results of our group show that the IFN $\gamma$-induced dysregulated murine phenotype depends on both, Stat 1 and SHH signaling (Walter et al., 2012). Thus, the lack of an important down-stream signal in the human situation might explain the species-dependent differences. As it is known that human gliomas and tumorigenic NSCs express SHH (Ehtesham et al., 2007) and comprise cells that simultaneously express neuronal and glial markers (Katsetos et al., 2001; Ignatova et al., 2002; Singh et al., 2003; Walton et al., 2009), this morphogene was associated with brain tumor formation and/or growth. Interestingly, in mice a link between IFN $\gamma$ and $\mathrm{SHH}$ signaling was observed as an ectopic expression of IFN $\gamma$ was shown to induce medulloblastoma formation via SHH overexpression (Lin et al., 2004). Our results in general point to the fact that, possibly fundamental differences in cytokine-induced signaling pathways between human and mouse lead to significant differences in the development of cellular phenotypes. Nevertheless, this type of fundamental differences can also be observed within a single genetic background if, for instance, merely the developmental age of a given cellular population differs. In this regard, several studies investigated the role of IFN $\gamma$ in human lymphocyte activation and found that, depending on their developmental age (neonatal vs. adult); IFN $\gamma$ induces divergent down-stream signaling pathways leading to significant differences in their ability to response to pathogens (Wilson et al., 1986; Marodi et al., 1994, 2001; Marodi, 2002). Discussed mechanisms for this divergent response of neonatal or adult lymphocytes to IFN $\gamma$ are a less effective Stat 1 phosphorylation in neonatal cells or an upregulation of a new class of cytokine signaling suppressors that can inhibit JAK-Stat pathway (Endo et al., 1997; Starr et al., 1997). In summary, our results admittedly reduce the relevance of an IFN $\gamma$-induced dysregulation of undifferentiated NSCs in a human genetic background. However, regarding the above mentioned considerations, it appears to be possible that human NSCs of a different developmental stage in comparison to those we used here can still react to IFN $\gamma$ exposure with an even misguided initiation of differentiation programs. Thus, our results can rather be interpreted in a way that a given cellular population in vitro, even on a human genetic background, might overemphasize results as it not reflects the complexity of an organism. This consideration is substantiated by observations from Johansson et al. (2008). In their study, immortalized hippocampal or striatal human neural stem/progenitor cells from 12-weeks-old fetal brains showed increased neurogenesis and MHC 1 expression after IFN $\gamma$ exposure during their differentiation phase without growth factors. The divergent results in comparison to our study might simply be explained by the developmental stage of the population during IFN $\gamma$ exposure as we used proliferating human NSCs under the influence of growth factors. That human fetal cells are principally able to coexpress GFAP and BIII-tubulin under non-inflammatory conditions in the ventricular and subventricular zones as well as under culture 
conditions was shown by Draberova et al. (2008). Probably this geno- and phenotypic rare case is only possible in a specific time frame of human fetal development and independent from inflammatory stimulation.

To further substantiate the relevance of IFN $\gamma$ during human CNS diseases, we verified the up-regulation of IFN $\gamma$ in the CNS. We demonstrate here that patient CSF contains elevated amounts of IFN $\gamma$ in comparison to control CSF of healthy individuals. Significantly elevated IFN $\gamma$ levels were found during relapses in multiple sclerosis $(n=3)$ and we also found increased levels in individual patients suffering from peripheral nervous plexus affections, Alzheimer dementia, viral myelitis, or stroke ( $n=1$, respectively). This is in accordance with the fact that human peripheral lymphocytes can secrete up to approximately 200-500 U/ml IFN $\gamma$ under neuroinflammatory conditions (Hirsch et al., 1985; Chan et al., 1991). As brain ependymal cells lack tight junctions, the CSF compartment in the brain ventricles exchanges neuroactive substances with the interstitial fluid of the brain parenchyma, including neurogenic zones (AlvarezBuylla and Lim, 2004; Shen et al., 2008; Tavazoie et al., 2008; Ming and Song, 2011; Hartung and Dihne, 2012). This might point to a possible anatomical relationship between IFN $\gamma$ accumulation within the CSF and NSCs within neurogenic zones, substantiating the relevance of IFN $\gamma$ effects on NSCs in humans. In summary, our results demonstrate that data collected on a murine genetic background cannot automatically be translated to the human situation and that even on human background results might differ depending on the developmental stage of the population and their maturation.

\section{MATERIALS AND METHODS CELL CULTURE}

The human NSCs used in this study were either derived from the human embryonic stem cell lines HUES 6 (hESC facility; Harvard University; Cambridge, MA, USA) or purchased in a neuroectodermal, predifferentiated form of the human embryonic stem cell line H9 (WiCell Research Institute, Madison, WI, USA/Invitrogen, Karlsruhe, Germany). The detailed neuronal differentiation protocol is described elsewhere (Dhara et al., 2008). Use of hESCs for derivation of neural progenitors was approved by the regulatory authorities at the Robert Koch Institute, Berlin, Germany, and derivation of neural progenitors was performed as follows: the hESCs were cultivated in knockout serum replacement (KSR) media (Cowan et al., 2004) on gelatin coated plates and a feeder layer of $\gamma$-irradiated mouse embryonic fibroblasts. Neural differentiation was performed by a protocol previously described by Lappalainen et al. (2010). Briefly, hESCs were dislodged and separated from the feeder cells and afterwards dissected into clusters that contained in between 1000 and 3500 cells. These clusters were placed in neural differentiation/proliferation media that consisted of 1:1 DMEM/F12 and Neurobasal media supplemented with $2 \mathrm{mM}$ GlutaMax, 1XB27, 1XN2, $0.25 \%$ BSA, $0.1 \mathrm{mM}$ non-essential amino acids, $25 \mathrm{U} / \mathrm{ml}$ penicillin/streptomycin (all products Invitrogen, Karlsruhe, Germany) and $20 \mathrm{ng} / \mathrm{ml}$ bFGF (Tebu-bio, Le Perray en Yvelines Cedex, France). Cells were propagated for $8-10$ weeks with a medium change every 2-3 days, the formed clusters were dissected once per week. After this time period, the vast majority of cells expressed NSC markers like nestin and Sox 2. Cells were then frozen. For experiments, human NSC were thawn and single cells were seeded on culture surfaces that were coated with poly-L-ornithine (PLO; 0.001\%; SigmaAldrich, Munich, Germany) and fibronectin $(20 \mu \mathrm{g} / \mathrm{ml}$; BioPur, Bubendorf, Switzerland). In parallel, we used the neuroectodermally pre-differentiated hNSC line (hNSC H9) for comparison. HNSC H9 cells were cultivated and propagated in the same way and in the same media as described above. Human NSCs derived from the cell line $\mathrm{H} 9$ were cultivated as free floating clusters and were dissected to single cells for experiments. The attached cells were either treated with human recombinant IFN $\gamma(1000 \mathrm{U} / \mathrm{ml}$; Millipore, Billerica, USA) or kept under control conditions for 3 days. This amount is equal to $100 \mathrm{ng} / \mathrm{ml}$ recombinant protein (murine and human). For differentiation experiments, cells had a day of recovery after seeding, then bFGF was withdrawn from the cells and differentiation was performed up to 3 months.

\section{IMMUNOCYTOCHEMISTRY}

For immunocytochemistry, cells were seeded on coated cover slips (VWR International, Darmstadt, Germany). After 3 days under the influence bFGF (20 ng/ml both Tebu-bio) and under different treatments as indicated, the cells were fixed with 4\% PFA (RotiHistofix, Carl Roth, Karlsruhe, Germany) for $15 \mathrm{~min}$ at room temperature. Cells were blocked for $30 \mathrm{~min}$ at room temperature with 1 fold Roti-Immuno-Block containing 0.25\% Triton X-100 for cell wall permeabilization (Carl Roth, Karlsruhe, Germany). Primary antibodies used at $4^{\circ} \mathrm{C}$ overnight were against $\beta$ IIItubulin (Tuj1; 1:500; R\&D Systems, Minneapolis, USA), glial fibrillary acid protein (GFAP) (1:500; Dako, Hamburg, Germany), Sox2 (1:50; R\&D Systems, Minneapolis, USA), IFN $\gamma$-R1 (1:500 Santa Cruz Biotechnology, Heidelberg, Germany), IFN $\gamma$-R2 (1:500; Santa Cruz Biotechnology, Heidelberg, Germany) and nestin (1:200; Covance, Munich, Germany). For detection of primary antibodies, fluoresceine-isothiocyanate (FITC; 1:500; Millipore, Billerica, USA) or indocarbocyanine (Cy3; 1:800; or Cy5; 1:200; Millipore, Billerica, USA) coupled secondary antibodies were used. The first and secondary antibodies were diluted in 1-fold Roti-Immuno-Block without Triton X-100 (Carl Roth, Karlsruhe, Germany). For visualization of cell nuclei cells were co-stained with DAPI (Invitrogen, Karlsruhe, Germany). For negative controls, primary antibodies were omitted in each experiment.

\section{MTT-ASSAY}

To analyze the population size of hNSCs, the optical density, indicative of conversion of 3-(4, 5-dimethylthiazol-2-yl)-2, 5-diphenyltetrazolium bromide (MTT; Sigma-Aldrich) into formazan crystals which takes place in live cells only, was determined after IFN $\gamma$ treatment at indicated concentrations. An OD value of 0.5 represents approximately 50,000 and an OD value of 1.0 represents approximately 100,000 live NSCs. The population size was measured after the indicated time intervals. Therefore, MTT (Invitrogen, Karlsruhe, Germany) was added to the cell culture media at a final concentration of $0.5 \mathrm{mg} / \mathrm{ml}$ after $5 \mathrm{~h}$ of incubation media was skipped and DMSO (Invitrogen, Karlsruhe, Germany) 
was added for the solubilization of formed crystals. We seeded the same amount of cells at day 0 and performed an MTT-Assay on day 3, 5, and 7. At every time point control cells and cells treated with human recombinant IFN $\gamma$ were tested. We used the same dose of human recombinant IFN $\gamma$, which was used in the murine study $(1000 \mathrm{U} / \mathrm{ml})$.

\section{REAL-TIME QUANTITATIVE PCR}

RNeasy Kit (Qiagen, Hilden, Germany) was used for RNA isolation of cultured NSCs. Then a reverse transcription into cDNA (ABI, Darmstadt, Germany) was performed. Real-time quantitative PCR was carried out by the usage of the 7500 fast or 7500 real-time quantitative PCR cycler (ABI, Darmstadt, Germany). SYBR green master mix (Qiagen, Hilden, Germany) or equivalent chemistry from another supplier (Quantace, London, UK) was used. The specific primers for genes of interest or the housekeeping gene were either purchased (QuantiTect primer assays, Qiagen) or self-designed (BioTEZ, Berlin, Germany). The genes of interest (target gene) in IFN $\gamma$-treated groups or control groups (PBS-treated) were analyzed in at least 3 independent cultures in triplicate each. Every experiment in IFN $\gamma$-treated or control (PBS-treated) groups provided delta CT values $(\Delta \mathrm{CT}$ : gene of interest minus reference gene).

\section{STATISTICAL ANALYSES}

Experiments were repeated with independent cultures at least three times in triplicate each. The resulting data sets were statistically analyzed und illustrated using the GraphPad Prism 4 (GraphPad Software Inc., San Diego, CA, USA, 2003) software. For approval of statistical significance between groups, a two-tailed unpaired $t$-test was performed. $P$-values $<0.05$ were considered to indicate significant differences.

\section{CSF COLLECTION AND IFN $\gamma$ ELISA}

Lumbar CSF was obtained from patients admitted to our department for diagnostic purposes. Patients suffered from multiple sclerosis (three samples), peripheral nervous plexus affections (one samples), Alzheimer's disease (one sample), viral myelitis (one sample), or stroke (one sample), were compared to control CSF (three samples). All CSF specimens were immediately centrifuged, aliquoted and stored at $-35^{\circ} \mathrm{C}$. Ethics approval for the use of human CSF was obtained from the institutional ethics committee. For IFN $\gamma$ detection in the CSF samples an IFN $\gamma$ ELISA by R\&D systems (R\&D systems, WiesbadenNordenstadt, Germany) was used. The experimental protocol followed the manufacturer's instructions. For statistical testing 3 control patients were compared with 3 MS patients with an unpaired two-tailed $t$-test.

\section{ACKNOWLEDGEMENTS}

We thank Christine Holland for excellent technical assistance and laboratory support. This work was supported by the Bundesministerium für Bildung und Forschung (BMBF, EuroTrans Bio, ESSENCE, 0315641B).

\section{REFERENCES}

Alvarez-Buylla, A., and Lim, D. A. (2004). For the long run: maintaining germinal niches in the adult brain. Neuron 41, 683-686.

Barker, R. A., Jain, M., Armstrong, R. J., and Caldwell, M. A. (2003). Stem cells and neurological disease. J. Neurol. Neurosurg. Psychiatry 74, 553-557.

Campbell, I. L. (2005). Cytokinemediated inflammation, tumorigenesis, and disease-associated JAK/STAT/SOCS signaling circuits in the CNS. Brain Res. Brain Res. Rev. 48, 166-177.

Chan, S. H., Perussia, B., Gupta, J. W., Kobayashi, M., Pospisil, M., Young, H. A., et al. (1991). Induction of interferon gamma production by natural killer cell stimulatory factor: characterization of the responder cells and synergy with other inducers. J. Exp. Med. 173, 869-879.

Cowan, C. A., Klimanskaya, I., McMahon, J., Atienza, J., Witmyer, J., Zucker, J. P., et al. (2004). Derivation of embryonic stem-cell lines from human blastocysts. $N$. Engl. J. Med. 350, 1353-1356.

Deng, W., Aimone, J. B., and Gage, F. H. (2010). New neurons and new memories: how does adult hippocampal neurogenesis affect learning and memory? Nat. Rev. Neurosci. 11, 339-350.

Deng, W., Saxe, M. D., Gallina, I. S., and Gage, F. H. (2009). Adultborn hippocampal dentate granule cells undergoing maturation modulate learning and memory in the brain. J. Neurosci. 29, 13532-13542.

De Simone, R., Levi, G., and Aloisi, F. (1998). Interferon gamma gene expression in rat central nervous system glial cells. Cytokine 10, 418-422.

Dhara, S. K., Hasneen, K., Machacek, D. W., Boyd, N. L., Rao, R. R., and Stice, S. L. (2008). Human neural progenitor cells derived from embryonic stem cells in feederfree cultures. Differentiation 76, 454-464.

Draberova, E., Del Valle, L., Gordon, J., Markova, V., Smejkalova, B., Bertrand, L., et al. (2008). Class III beta-tubulin is constitutively coexpressed with glial fibrillary acidic protein and nestin in midgestational human fetal astrocytes: implications for phenotypic identity. J. Neuropathol. Exp. Neurol. 67, 341-354.

Ehtesham, M., Sarangi, A., Valadez, J. G., Chanthaphaychith, S., Becher,
M. W., Abel, T. W., et al. (2007). Ligand-dependent activation of the hedgehog pathway in glioma progenitor cells. Oncogene 26, 5752-5761.

Endo, T. A., Masuhara, M., Yokouchi, M., Suzuki, R., Sakamoto, H., Mitsui, K., et al. (1997). A new protein containing an $\mathrm{SH} 2$ domain that inhibits JAK kinases. Nature 387, 921-924.

Farrar, M. A., and Schreiber, R. D. (1993). The molecular cell biology of interferon-gamma and its receptor. Annu. Rev. Immunol. 11, 571-611.

Gogel, S., Gubernator, M., and Minger, S. L. (2011). Progress and prospects: stem cells and neurological diseases. Gene. Ther. 18, 1-6.

Griffin, D. E. (1997). Cytokines in the brain during viral infection: clues to HIV-associated dementia. J. Clin. Invest. 100, 2948-2951.

Harrower, T., and Barker, R. A. (2005). Cell therapies for neurological disease - from bench to clinic to bench. Expert Opin. Biol. Ther. 5, 289-291.

Hartung, H. P., and Dihne, M. (2012). Volume transmission-mediated encephalopathies: a possible new concept? Arch. Neurol. 69, 315-321.
Hirsch, R. L., Panitch, H. S., and Johnson, K. P. (1985). Lymphocytes from multiple sclerosis patients produce elevated levels of gamma interferon in vitro. J. Clin. Immunol. 5, 386-389.

Ignatova, T. N., Kukekov, V. G., Laywell, E. D., Suslov, O. N., Vrionis, F. D. and Steindler, D. A. (2002). Human cortical glial tumors contain neural stem-like cells expressing astroglial and neuronal markers in vitro. Glia 39, 193-206.

Illes, S., Theiss, S., Hartung, H. P., Siebler, M., and Dihne, M. (2009). Niche-dependent development of functional neuronal networks from embryonic stem cell-derived neural populations. BMC Neurosci. 10:93. doi: 10.1186/1471-2202-10-93

Johansson, S., Price, J., and Modo, M. (2008). Effect of inflammatory cytokines on major histocompatibility complex expression and differentiation of human neural stem/progenitor cells. Stem Cells 26, 2444-2454.

Katsetos, C. D., Del Valle, L., Geddes, J. F., Assimakopoulou, M., Legido, A. Boyd, J. C., et al. (2001). Aberrant localization of the neuronal class III beta-tubulin in astrocytomas. Arch. Pathol. Lab. Med. 125, 613-624. 
Kempermann, G. (2002). Neuronal stem cells and adult neurogenesis. Ernst Schering Res. Found. Workshop 7-28.

Kempermann, G. (2011). Seven principles in the regulation of adult neurogenesis. Eur. J. Neurosci. 33, 1018-1024.

Kim, I. J., Beck, H. N., Lein, P. J., and Higgins, D. (2002). Interferon gamma induces retrograde dendritic retraction and inhibits synapse formation. J. Neurosci. 22, 4530-4539.

Kim, S. J., Son, T. G., Kim, K., Park, H. R., Mattson, M. P., and Lee, J. (2007). Interferongamma promotes differentiation of neural progenitor cells via the JNK pathway. Neurochem. Res. 32, 1399-1406.

Komatsu, T., Bi, Z., and Reiss, C. S. (1996). Interferon-gamma induced type I nitric oxide synthase activity inhibits viral replication in neurons. J. Neuroimmunol. 68, 101-108.

Lappalainen, R. S., Salomaki, M., Yla-Outinen, L., Heikkila, T. J., Hyttinen, J. A., Pihlajamaki, H., et al. (2010). Similarly derived and cultured hESC lines show variation in their developmental potential towards neuronal cells in long-term culture. Regen. Med. 5, 749-762.

Lehtonen, A., Matikainen, S., and Julkunen, I. (1997). Interferons up-regulate STAT1, STAT2, and IRF family transcription factor gene expression in human peripheral blood mononuclear cells and macrophages. J. Immunol. 159, 794-803.

Lin, W., Kemper, A., McCarthy, K. D., Pytel, P., Wang, J. P., Campbell, I. L., et al. (2004). Interferon-gamma induced medulloblastoma in the developing cerebellum. J. Neurosci. 24, 10074-10083.

Makela, J., Koivuniemi, R., Korhonen, L., and Lindholm, D. (2010). Interferon-gamma produced by microglia and the neuropeptide PACAP have opposite effects on the viability of neural progenitor cells. PLoS ONE 5:e11091. doi: 10.1371/journal.pone.0011091

Marodi, L. (2002). Deficient interferongamma receptor-mediated signaling in neonatal macrophages. Acta Paediatr. Suppl. 91, 117-119.
Marodi, L., Goda, K., Palicz, A., and Szabo, G. (2001). Cytokine receptor signalling in neonatal macrophages: defective STAT-1 phosphorylation in response to stimulation with IFN-gamma. Clin. Exp. Immunol. 126, 456-460.

Marodi, L., Kaposzta, R., Campbell, D. E., Polin, R. A., Csongor, J., and Johnston, R. B., Jr. (1994). Candidacidal mechanisms in the human neonate. Impaired IFNgamma activation of macrophages in newborn infants. J. Immunol. 153, 5643-5649.

Ming, G. L., and Song, H. (2011). Adult neurogenesis in the mammalian brain: significant answers and significant questions. Neuron 70, 687-702.

Ousman, S. S., Wang, J., and Campbell, I. L. (2005). Differential regulation of interferon regulatory factor (IRF)-7 and IRF-9 gene expression in the central nervous system during viral infection. J. Virol. 79, 7514-7527.

Rady, P. L., Cadet, P., Bui, T. K., Tyring, S. K., Baron, S., Stanton, G. J., et al. (1995). Production of interferon gamma messenger RNA by cells of non-immune origin. Cytokine 7 , 793-798.

Ramana, C. V., Grammatikakis, N., Chernov, M., Nguyen, H., Goh, K. C., Williams, B. R., et al. (2000). Regulation of cmyc expression by IFN-gamma through Statl-dependent and independent pathways. EMBO J. 19, 263-272.

Schreiber, R. D., and Farrar, M. A. (1993). The biology and biochemistry of interferon-gamma and its receptor. Gastroenterol. Jpn. 28(Suppl. 4), 88-94. discussion: 95-96.

Schreiber, R. D., Farrar, M. A., Hershey, G. K., and Fernandez-Luna, J. (1992). The structure and function of interferon-gamma receptors. Int. J. Immunopharmacol. 14, 413-419.

Schroder, K., Hertzog, P. J., Ravasi, T., and Hume, D. A. (2004). Interferon-gamma: an overview of signals, mechanisms and functions. J. Leukoc. Biol. 75, 163-189.

Shen, Q., Wang, Y., Kokovay, E., Lin, G., Chuang, S. M., Goderie, S. K., et al. (2008). Adult SVZ stem cells lie in a vascular niche: a quantitative analysis of niche cell-cell interactions. Cell Stem Cell 3, 289-300.

Singh, S. K., Clarke, I. D., Terasaki, M., Bonn, V. E., Hawkins, C., Squire, J., et al. (2003). Identification of a cancer stem cell in human brain tumors. Cancer Res. 63, 5821-5828.

Starr, R., Willson, T. A., Viney, E. M., Murray, L. J., Rayner, J. R., Jenkins, B. J., et al. (1997). A family of cytokine-inducible inhibitors of signalling. Nature 387, 917-921.

Sweeten, T. L., Posey, D. J., Shankar, S., and McDougle, C. J. (2004). High nitric oxide production in autistic disorder: a possible role for interferon-gamma. Biol. Psychiatry 55, 434-437.

Tavazoie, M., Van der Veken, L., SilvaVargas, V., Louissaint, M., Colonna, L., Zaidi, B., et al. (2008). A specialized vascular niche for adult neural stem cells. Cell Stem Cell 3, 279-288.

Temple, S. (2001). The development of neural stem cells. Nature 414, 112-117.

Temple, S., and Qian, X. (1996). Vertebrate neural progenitor cells: subtypes and regulation. Curr. Opin. Neurobiol. 6, 11-17.

Walter, J., Hartung, H.-P., and Dihné, M. (2012). Interferon gamma and sonic hedgehog signaling are required to dysregulate murine neural stem/precursor cells. PLOS ONE 7:e43338. doi: 10.1371/journal.pone.0043338

Walter, J., Honsek, S. D., Illes, S. Wellen, J. M., Hartung, H. P., Rose, C. R., et al. (2011). A new role for interferon gamma in neural stem/precursor cell dysregulation. Mol. Neurodegener. 6, 18.

Walton, N. M., Snyder, G. E., Park, D., Kobeissy, F., Scheffler, B., and Steindler, D. A. (2009). Gliotypic neural stem cells transiently adopt tumorigenic properties during normal differentiation. Stem Cells 27, 280-289.

Wang, J., Lin, W., Popko, B., and Campbell, I. L. (2004). Inducible production of interferon-gamma in the developing brain causes cerebellar dysplasia with activation of the Sonic hedgehog pathway. Mol. Cell. Neurosci. 27, 489-496.

Wang, Y., Imitola, J., Rasmussen, S., O'Connor, K. C., and Khoury, S. J. (2008). Paradoxical dysregulation of the neural stem cell pathway sonic hedgehog-Glil in autoimmune encephalomyelitis and multiple sclerosis. Ann. Neurol. 64, 417-427.

Wei, Y. P., Kita, M., Shinmura, K. Yan, X. Q., Fukuyama, R., Fushiki, S., et al. (2000). Expression of IFN-gamma in cerebrovascular endothelial cells from aged mice. J. Interferon Cytokine Res. 20, 403-409.

Whitney, N. P., Eidem, T. M., Peng, H., Huang, Y., and Zheng, J. C. (2009). Inflammation mediates varying effects in neurogenesis: relevance to the pathogenesis of brain injury and neurodegenerative disorders. J. Neurochem. 108, 1343-1359.

Wilson, C. B., Westall, J., Johnston, L., Lewis, D. B., Dower, S. K., and Alpert, A. R. (1986). Decreased production of interferon-gamma by human neonatal cells. Intrinsic and regulatory deficiencies. J. Clin. Invest. 77, 860-867.

Yla-Outinen, L., Heikkila, J., Skottman, H., Suuronen, R., Aanismaa, R., and Narkilahti, S. (2010). Human cell-based micro electrode array platform for studying neurotoxicity. Front. Neuroeng. 3:111. doi: 10.3389/fneng.2010.00111

Conflict of Interest Statement: The authors declare that the research was conducted in the absence of any commercial or financial relationships that could be construed as a potential conflict of interest.

Received: 01 March 2012; accepted: 17 October 2012; published online: 08 November 2012.

Citation: Walter J and Dihné M (2012) Species-dependent differences of embryonic stem cell-derived neural stem cells after Interferon gamma treatment. Front. Cell. Neurosci. 6:52. doi: 10.3389/ fncel.2012.00052

Copyright (c) 2012 Walter and Dihné. This is an open-access article distributed under the terms of the Creative Commons Attribution License, which permits use, distribution and reproduction in other forums, provided the original authors and source are credited and subject to any copyright notices concerning any third-party graphics etc. 